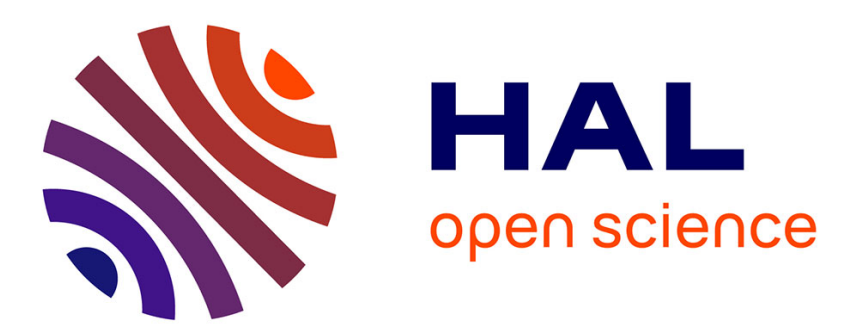

\title{
Investigating the influence of the operating frequency on the gas phase emitter effect of dysprosium in ceramic metal halide lamps
}

\author{
J Reinelt, M Westermeier, C Ruhrmann, A Bergner, G M J F Luijks, P
}

Awakowicz, J Mentel

\section{To cite this version:}

J Reinelt, M Westermeier, C Ruhrmann, A Bergner, G M J F Luijks, et al.. Investigating the influence of the operating frequency on the gas phase emitter effect of dysprosium in ceramic metal halide lamps. Journal of Physics D: Applied Physics, 2011, 44 (22), pp.224006. 10.1088/0022-3727/44/22/224006 . hal-00620592

\section{HAL Id: hal-00620592 \\ https://hal.science/hal-00620592}

Submitted on 8 Sep 2011

HAL is a multi-disciplinary open access archive for the deposit and dissemination of scientific research documents, whether they are published or not. The documents may come from teaching and research institutions in France or abroad, or from public or private research centers.
L'archive ouverte pluridisciplinaire $\mathbf{H A L}$, est destinée au dépôt et à la diffusion de documents scientifiques de niveau recherche, publiés ou non, émanant des établissements d'enseignement et de recherche français ou étrangers, des laboratoires publics ou privés. 


\title{
Investigating the influence of the operating frequency on the gas phase emitter effect of dysprosium in ceramic metal halide lamps
}

\author{
J Reinelt ${ }^{1,2}$, M Westermeier ${ }^{1,2}$, C Ruhrmann ${ }^{1}$, A Bergner ${ }^{1}$, \\ G M J F Luijks ${ }^{3}, \mathbf{P}$ Awakowicz $^{1}$ and $\mathrm{J}_{\text {Mentel }^{1}}$ \\ ${ }^{1}$ Ruhr University Bochum, Electrical Engineering and Plasma Technology, D-44780 \\ Bochum, Germany \\ 2 Present address: RWE Effizienz GmbH, Freistuhl 7, D-44137 Dortmund, Germany \\ ${ }^{3}$ Philips Lighting, GBU HID, P.O.box 80020, 5600JM Eindhoven, The Netherlands \\ E-mail: juergen.mentel@ruhr-uni-bochum.de
}

\begin{abstract}
The dependence of the gas phase emitter effect of $D y$ on a variation of the operating frequency between some $\mathrm{Hz}$ and $2 \mathrm{kHz}$ is investigated in a HID lamp. The buffer gas of the lamp consisting of $\mathrm{Ar}, \mathrm{Kr}$ and predominantly $\mathrm{Hg}$ is seeded with $\mathrm{Dy} I_{3}$, its burner vessel is formed from transparent YAG material. Phase and spatial resolved emission spectroscopy in front of the lamp electrode and pyrometric temperature measurements along the tungsten electrode are performed with a spectroscopic set up. $D y$ atom and ion densities in front of the electrode are deduced from absolute intensities of optically thin $D y$ lines and a plasma temperature, derived from the absolute intensity of mercury lines. Phase resolved values of the electrode tip temperature $T_{t i p}$ and input power $P_{i n}$ are obtained from temperature distributions along the electrode. Distinctly higher $D y$ ion and atom densities are measured in front of the electrode within the cathodic phase. With increasing operating frequency a reduction of both, atoms and ions, is observed in front of the cathode. In contrast, an increase of the ion density in front of the anode is seen. Moreover, the $D y$ ion density is drastically reduced by an additional seeding of the lamp with $T l I$. It is found that an up rating of the $D y$ ion density is correlated with a decline of $T_{t i p}$ and $P_{i n}$. At higher frequencies this effect takes place not only within the cathodic phase but also within the anodic phase. The reduction of the average electrode tip temperature on the order of several hundred Kelvin compared to a YAG lamp with a pure mercury filling is explained by a $D y$ monolayer on the electrode surface which is sustained by a $D y$ ion current.
\end{abstract}

Submitted to: J. Phys. D: Appl. Phys. 


\section{Introduction}

Today the most effective point shaped light sources to generate visible light for outdoor applications in the range of k-lumens are high intensity discharge (HID) lamps, e.g. high pressure sodium lamps. They are characterized by a high efficacy on the order of $100-150 \mathrm{~lm} / \mathrm{W}$, a long life time on the order of $10000-25000 \mathrm{~h}$ and low costs. In addition white light and a high colour rendering can be achieved with a special version of HID lamps: the metal halide (MH) lamps. In these lamps a buffer gas is seeded together with carefully chosen mixes of metal iodides. They can provide rich emission spectra by which white light with high colour quality can be created. A high metal vapour pressure is favourable for a high colour rendering. It is determined by the lowest value of the temperature on the inner wall of the lamp tube, the so called cold spot temperature. To make the cold spot temperature as high as possible the tubes of $\mathrm{MH}$ lamps are preferably made of ceramic material, e.g. translucent alumina. It substitutes fused silica which was used previously since it permits higher wall loadings and higher temperatures. As a result ceramic MH lamps are characterized not only by high efficacies and long life times but also by excellent colour rendering indices on the order of 90. For this reason they are increasingly adopted for indoor applications such as shop lighting.

The high demands on the load capacity of the electrodes are one of the main problems with HID lamps. The tip temperature of electrodes made of pure tungsten may increase up to its melting point, $T=3695 \mathrm{~K}$, to supply a sufficiently high current density for a diffuse attachment of the lamp arc on the electrodes $[1,2,3,4]$. But the electrode temperature can substantially be reduced by lowering the work function of the electrode material making use of the so called emitter effect. It is generated by a dipole layer being formed by a monolayer of electropositive atoms on the tungsten surface. It reduces the potential barrier at the electrode surface for electrons leaving or entering the electrode.

Several techniques may be used to form a dipole layer of electropositive atoms on the hot tungsten electrode.

A thorium monolayer may be generated by doping the tungsten electrodes with $\mathrm{ThO}_{2}$ by an amount of several percent of the electrode volume [5]. A reduction in the effective work function of HID cathodes was verified by pyrometric measurements of the electrode temperature and by probe measurements of the cathode fall in the model lamp $[6,7,8]$. It was also shown by measurements in the model lamp that doping of a tungsten electrode with $\mathrm{ThO}_{2}$ does not change the anode temperature. It demonstrates that the effective work function of the thoriated anode is comparable to that of a pure tungsten anode $[9,10]$.

Another approach to forming a dipole layer is to deposit emitter material, e.g. a 
Ba-compound $\left(\mathrm{Ba}_{2} \mathrm{CaWO}_{6}\right.$ or $\left.\mathrm{Ba}_{3} Y_{2} W_{9}\right)$ within the interspaces of a closely wound tungsten coil at some distance from the electrode tip. When the electrode is heated up the compound is dissociated and the $B a$ emitter diffuses along the electrode surface to the tip [5], a theory, which was recently confirmed by measurements [11].

A quite versatile approach is the deposition of an atomic monolayer on the electrode surface from the gas phase being called "gas phase emitter effect" [12]. A reduction of the work function by a sodium monolayer on the cathode and anode of a high pressure sodium (HPS) lamp could be demonstrated if appropriate lamp parameters were chosen $[13,14]$. A monolayer can be realized with a large variety of elements by adding appropriate metal iodides to the lamp filling. They are vaporized and dissociated during lamp operation so that a partial pressure of metal vapour is formed within the lamp tube. A gas phase emitter effect induced by a $T h I_{4}$ additive was demonstrated already many years ago in a high pressure mercury lamp operated with an ac current [15]. But it is not clear whether the emitter effect is limited to the cathodic half period or is also effective within the anodic half period.

A gas phase emitter effect can also be realized if a HID metal halide lamp is seeded with rare earth iodides. A detailed investigation of this effect in a ceramic metal halide lamp operated with a dc current in a mercury atmosphere seeded with different mixtures of $\mathrm{NaI}, \mathrm{TlI}$ and $\mathrm{DyI}_{3}$ is given in [12]. A transparent ceramic YAG tube was used to allow electrode temperature measurements. A distinct reduction in the work function at the cathode was demonstrated by a measured drastic reduction of the cathode temperature. At the anode a minor or even doubtful emitter effect was found by measurements of the electrode tip temperature. If the lamp is operated with a switched dc current an increase of the average electrode tip temperature $T_{t i p}$ within the cathodic phase was found by reason of anode heating during the preceding anodic half period. However, the averaged temperature remains below the value for a lamp with a pure mercury filling $[16,17]$. By spectroscopic measurements of the $D y$ atom and ion density it was shown that a decrease of the electrode temperature is correlated with an increased $D y$ atom and ion density in front of the electrode [16].

This observation has brought up the idea that the $D y$ layer on the electrode is mainly formed by a $D y$ ion current towards the electrode during the cathodic phase. A generation of the emitter effect by an ion current may explain why an additional seeding of the YAG lamp with sodium seems to hamper the emitter effect of $D y$ [16]. In this case predominantly a $\mathrm{Na}$ ion current in front of the cathode is formed since the ionisation energy of $N a\left(E_{i}=5.139 \mathrm{eV}\right)$ is lower and its mass $\left(A_{r}=23\right)$ smaller than that of $D y\left(E_{i}=5.93 \mathrm{eV}, A_{r}=162.5\right)$. But the adsorption energy $E_{a}$ of $N a$ on a tungsten surface $\left(E_{a}=2.33 \mathrm{eV}\right)$ is distinctly smaller than that of $D y\left(E_{a}=4.97 \mathrm{eV}\right)$ so that it is, different to $D y$, less appropriate to form a stable monolayer on a hot electrode within a MH lamp [18, 19, 20]. 
The importance of an ion current of the emitter material to the cathode is also indicated by an advancement of the spot attachment with an increasing $D y$ density $[12,16,21]$. The shape of the spot induced by a high $D y$ density differs from the spot mode investigated in great detail in $[1,3,22,23,24,25,26]$. The spot attachment induced by the gas phase emitter effect should therefore be called "emitter spot". The statement in [27] that the emitter effect favours the formation of a diffuse mode obviously does not apply to the emitter spot.

The behaviour of tungsten electrodes for HID lamps was investigated recently in the so called Bochum model lamp filled with argon [6] in dependence on the operating frequency being varied between $10 \mathrm{~Hz}$ and $10 \mathrm{kHz}$ [28]. The Bochum model lamp is formed by a quartz tube, sealed vacuum-tight by two metallic end pieces with feedthroughs containing movable holders for the tungsten electrodes. The measuring results have shown that the cathodic phase becomes more and more dominant with increasing operating frequency. It was found that with increasing operating frequency the electrode temperature approaches the value of the cathode at dc operation, while the influence of the anodic phase is pushed back. The same may happen in MH lamps, in which the gas phase emitter effect is heavily dependent on the electrode polarity.

To check this speculation the electrodes of YAG lamps and the plasma in front of them are investigated in dependence on the operating frequency being varied between $1 \mathrm{~Hz}$ and $2 \mathrm{kHz}$. Phase resolved pyrometric measurements of the electrode temperature and phase resolved measurements of the $D y$ atom and ion density are accomplished by emission spectroscopy. Appropriate measuring methods are already given in previous papers $[16,28]$ so that only a short sketch will be given. YAG lamps with different fillings and electrodes with different diameters are operated with a switched dc current of constant amplitude but different operating frequencies. Initially, as a reference, phase resolved electrode tip temperatures and plasma temperatures in front of the electrode are given for a YAG lamp with a pure mercury filling. The plasma temperatures are deduced from the absolute intensity of mercury lines. In a next step YAG lamps, in which the buffer gas mercury is only seeded with $D y I_{3}$, are investigated for electrodes with different diameters. To clarify the role of thallium in $\mathrm{MH}$ lamps some measurements of YAG lamps are given, which are seeded with a mixture of $T l I$ and $D y I_{3}$. The major part of this investigation extends measurements to higher frequencies which were already made at low operating frequencies [16]. The paper is to a large extent an excerpt of Reinelt's PhD thesis [29]. 


\section{Experimental setup}

\subsection{YAG lamp}

The HID lamps under investigation have a special research design being given in detail in [12]. A burner vessel with an ovoid shape is formed from yttrium-alumina-garnet (YAG), a transparent material, which allows optical observation. Tungsten rod electrodes are mounted on a $M o$ feedthrough for ceramic lamps and sealed in the lamps using a sealing ceramic. The electrodes have a diameter of $d=360,450$ and $500 \mu \mathrm{m}$ and an interelectrode spacing of $g=7 \mathrm{~mm}$. The electrodes extend $5 \mathrm{~mm}$ into the tube, their total length is $20-25 \mathrm{~mm}$. The burner is enveloped by an outer glass bulb furnished with a lamp base. The lamp is filled with an argon-krypton mixture as buffer gas at a pressure of $p=30 \mathrm{kPa}$ for lamp ignition and in addition with an amount of mercury that produces an unsaturated mercury background pressure of $p=2 \mathrm{MPa}$ [12]. Some lamps are seeded with $D y I_{3}$, another one with a mixture of $D y I_{3}$ and $T l I$. For comparison also lamps without any salt additive are investigated. The properties of lamps for which measurements are presented are listed in table 1. The lamps are designed for an effective electrical power of $P_{e l}=70 \mathrm{~W}$. The lamps are operated in a vertical position with the cold spot and the salt pool associated with it on the lower end of the lamp. The optical measurements are performed at the upper electrode since at the lower electrode the transparency of the tube wall is affected by the liquid salt pool.

\begin{tabular}{lllllll}
\hline $\begin{array}{l}\text { Lamp } \\
\text { type }\end{array}$ & $\begin{array}{l}\text { Electrode } \\
\text { diameter } \\
(\mu \mathrm{m})\end{array}$ & $\begin{array}{l}\text { Electrode } \\
\text { length } \\
(\mathrm{mm})\end{array}$ & $\begin{array}{l}\mathrm{Hg} \\
(\mathrm{mg})\end{array}$ & $\begin{array}{l}\text { Total } \\
\text { salt } \\
(\mathrm{mg})\end{array}$ & $\begin{array}{l}\mathrm{Tl} \\
(\mathrm{mol} \%)\end{array}$ & $\begin{array}{l}\mathrm{Dy} \\
(\mathrm{mol} \%)\end{array}$ \\
\hline $\mathrm{Hg}$ & $d=360$ & $l=5$ & 6 & 0 & 0 & 0 \\
$\mathrm{D}$ & $d=360$ & $l=5$ & 6 & 1 & 0 & 100 \\
$\mathrm{D}$ & $d=450$ & $l=5$ & 6 & 1 & 0 & 100 \\
$\mathrm{D}$ & $d=500$ & $l=5$ & 6 & 1 & 0 & 100 \\
$\mathrm{TD}$ & $d=450$ & $l=5$ & 6 & 2 & 50 & 50 \\
\hline
\end{tabular}

Table 1. Properties of the lamps investigated in this work

The YAG lamp is operated with a current source (Feucht Elektronik DCU/I 225030) being controlled by the output voltage of a signal generator. It is able to provide $\mathrm{dc}$, ac, pulsed or transient currents of arbitrary waveform up to frequency of $30 \mathrm{kHz}$, a current amplitude of several amps and a maximum open-circuit voltage of $400 \mathrm{~V}$.

Standing acoustic oscillations may occur in MH lamps within various frequency regions. The YAG lamps being investigated here are undisturbed by acoustic resonances in the following frequency bands:

$$
f \leq 3500 \mathrm{~Hz}
$$


Investigating the influence of the operating frequency on the gas phase emitter effect of Dy6

$$
\begin{aligned}
& f=23.2 \mathrm{kHz} \\
& f \geq 300 \mathrm{kHz}
\end{aligned}
$$

\subsection{Measuring system for phase resolved emission spectroscopy}

Almost the same spectroscopic measuring system is used for phase resolved measurements of the electrode temperature and of the particle densities in front of the electrode. Only some minor modifications are needed. The experimental setup was already described in $[16,25]$, so that a sketch will be sufficient here.

The spectrograph in use is a $250 \mathrm{~mm}$ apparatus from Chromex with an aperture ratio of $f / 4.0$. A grating is used mostly with a line density of $1200 \mathrm{~mm}^{-1}$. The entrance slit is adjusted for pyrometric measurements to a width of $100 \mu \mathrm{m}$ and in the case of spectroscopic measurements to $25 \mu \mathrm{m}$. To record the emission of the lamp by the spectrograph an optical imaging system is mounted in between. A telecentric imaging system consisting of two achromats projects an image of the lamp onto the entrance plane of the spectrograph with a magnification of $M=1$. A Dove prism between the two achromats is used to adjust either the image of the electrode parallel or the discharge axis perpendicular to the vertical entrance slit of the spectrograph.

The output of the spectrograph is recorded with a CCD camera SENSICAM QE from PCO mounted in the image plane of it. The camera resolution along the entrance slit is 1376 pixel and along the wavelength axis 1040 pixel. The pixel size amounts to $6.45 \mu \mathrm{m} \times 6.45 \mu \mathrm{m}$. It determines the spatial resolution along the entrance slit of the spectrograph. The camera offers the possibility of an internal binning summing up the counts of a defined number of adjacent pixel. The dynamic range of the camera is 14 bits which implies a maximum count rate of 4096 counts per pixel. The exposure time of the camera $t_{\exp }$ can be varied between $500 \mathrm{~ns}$ and $3600 \mathrm{~s}$. The CCD chip of the camera is sensitive within the wavelength interval from 280 to $1100 \mathrm{~nm}$. A calibration of the optical system in absolute values of radiance is needed for the measurements at the electrode and at the plasma. For this purpose a tungsten ribbon lamp (Wi17g3 from OSRAM) with a known radiance in dependence on wavelength for a specified current is positioned on the same place as the lamp under investigation.

The temporal resolution of the camera is limited by the read out time of the CCD chip, which amounts approximately to $90 \mathrm{~ms}$. This means that for operating frequencies above $f>5 \mathrm{~Hz}$ not more than one measurement within one half period can be made. Therefore repeated phase resolved measurements are needed to get information about the time-evolution of phenomena of interest within the half period. The substitution of a temporal by a phase resolution does not raise a problem as long as the lamp operation is really periodic. In this case only a reproducible periodical trigger signal is required 
as a starting point for a series of successively phase delayed exposures.

The delay time of the current source, which is realized by an electronic device, varies too much in dependence on the operating parameters that the "sync" output of the signal generator, by which the current source is controlled, is suitable to provide an appropriate trigger pulse. Thus the reference phase has to be generated on the output side of the amplifier. The current zero crossing is inapplicable since the current may not rise fast enough after current zero crossing to be able to generate a well defined trigger pulse. Therefore the trigger signal is deduced from the voltage zero crossing of the lamp [24]. To trigger the camera only the rising edge of the voltage signal is used. The uncertainty of the trigger pulse does not exceed $2 \mu \mathrm{s}$. To take pictures at different phase angles $\varphi$ a delay generator (STANFORD RESEARCH SYSTEM DG 535) is used to shift the trigger signal from voltage zero crossing. A block diagram of the trigger set up is already given in [25].

\section{Pyrometric electrode temperature measurement}

It is favorable for the pyrometric measurements to use a segment of the spectrum emitted by the electrode, which is as far as possible in the infrared region but still in the sensitivity range of the CCD camera. Furthermore, the plasma radiation emitted by the YAG lamps should be negligible compared to the emission of the electrode surface. A suitable wavelength for temperature measurements of the electrodes in the YAG lamps is $\lambda=718 \mathrm{~nm}$. An edge filter with an edge wavelength $\lambda_{\text {edge }}=600 \mathrm{~nm}$ is used to suppress all radiation with a wavelength below $\lambda_{\text {edge }}$. It prevents a falsification of the measurement by the second order spectrum produced by the grating emitted at $\lambda_{\text {meas }} / 2$. The integral transmission $\mathrm{Tr}$ of the YAG tube and of the outer glass bulb which has to be taken into account amounts to $\operatorname{Tr} \approx 72 \%$ at $\lambda_{\text {meas }}=718 \mathrm{~nm}$.

A spatial profile of the spectral radiance $I_{e l}$ of the electrode at $\lambda=718 \mathrm{~nm}$ along the electrode length within the tube is recorded by a single measurement at a selected phase angle $\varphi$. A grey body radiator is assumed to determine axial temperature profiles $T(z, \varphi)$ from the measured radiances $I_{e l}(z, \varphi)$. The spectral radiance of the grey body $I_{g b}(\lambda, T)$ is given by Planck's law and by temperature and wavelength dependent spectral emissivity $\epsilon(\lambda, T)<1$. An inspection of literature [30] and a comparison between measurements and simulation ([3] figure 14) showed that the spectral emissivities of de Vos [31] are the most trustable ones. Spatial profiles $T(z, \varphi)$ are determined comparing measured values $I_{e l}(z, \varphi)$ with $I_{g b}(T, \lambda=718 \mathrm{~nm})$ calculated in dependence on $T$. The accuracy of the temperature measurements especially in the vicinity of the electrode tip is mainly affected by questionable values of $\epsilon$. They are in general exaggerated in an undefined manner by an enhanced surface roughness being produced by the arc attachment at the electrode tip. The exaggerated values of $\epsilon$ increase the radiance at the electrode tip so that the comparison with the calculated values of $I_{g b}(T)$ delivers too 
high temperature values at the electrode tip $T_{t i p}$.

Reliable values of $T_{t i p}$ were obtained in [6] for electrodes operated with a dc current by matching measured temperature distributions $T(z)$ within a region of confidence between a lower temperature limit $T_{l}$ and an upper temperature limit $T_{u p}<T_{\text {tip }}$ by a least square fit procedure to numerical solutions of the one dimensional heat balance of the electrode. The electrode tip temperature $T_{t i p}$ and the total power input into the electrode $P_{i n}$ were determined by extrapolating the solutions up to the electrode tip. But it was shown in [28] that the method becomes questionable if in the case of an ac operation the heat capacity of the electrode has an influence on the temperature distribution. A distinct variation of the temperature with $\varphi$ occurs only in the vicinity of the electrode tip, but disappears with increasing distance from the tip [25]. Its extension is given by the penetration depth of a sinusoidal modulation of the temperature at the electrode tip

$$
\lambda_{\text {pen }}=\left(\frac{\kappa}{\rho_{M} c_{p} 2 \pi f}\right)^{1 / 2}
$$

where $\kappa(T)$ is the thermal conductivity of tungsten in dependence on temperature, $\rho_{M}$ the mass density of tungsten and $c_{p}(T)$ its heat capacity per unit mass. A comprehensive presentation of the data is given in [30]. $\lambda_{\text {pen }}$ decreases with $f^{-1 / 2}$ and is already less than $1 \mathrm{~mm}$ for $f=10 \mathrm{~Hz}$ and $T=3000 \mathrm{~K}$. This means that $T_{\text {tip }}(\varphi)$ can, different to [6], only be determined with an appropriate phase resolution if the temperature distribution in the vicinity of the electrode tip can be taken into account.

For this purpose the time dependent heat balance of the electrode was considered in [28]. A rotational symmetric electrode temperature distribution is assumed as it is realized by a diffuse attachment of an arc on the electrode. Additionally the $r$ dependence of $T$ along the electrode rod is neglected. A thin disk of the electrode of thickness $d z$ in a distance $z$ from the electrode tip and a change of the phase angle $d \varphi$ is considered to calculate the power balance of the electrode in dependence on time/phase. Since during the exposure time $t_{e}=\omega^{-1} \varphi_{e}$ of the camera $\left(\frac{\partial T}{\partial t}\right)_{t_{e}}=\omega\left(\frac{\partial T}{\partial \varphi}\right)_{\varphi_{e}}$ is almost constant, the spatial derivative $\partial T / \partial z$ can be substituted by the total derivative $d T / d z$. Thus, the heat balance of the electrode disk can be written as:

$$
r_{E}^{2} \pi \frac{d}{d z}\left(\kappa(T) \frac{d T}{d z}\right)=-2 \pi r_{E} q_{E}(T)+2 \pi r_{E} \epsilon_{t o t} \sigma_{S B} T^{4}+r_{E}^{2} \pi \frac{\kappa}{\lambda_{p e n}^{2}}\left(\frac{\partial T}{\partial \varphi}\right)_{\varphi_{e}},
$$

where $r_{E}$ is the electrode radius, $q_{E}(T)$ the power input per unit area from the plasma into the cylinder barrel of the rod, $\epsilon_{t o t}$ the total emissivity of tungsten and $\sigma_{S B}$ the Stefan-Boltzmann constant. Data for $\epsilon_{t o t}$ are given in [30]. An analytic solution of the differential equation was given in [28]:

$$
z(T)=
$$


Investigating the influence of the operating frequency on the gas phase emitter effect of Dy9

$$
\int_{T^{\prime}=T}^{T_{\text {tip }}} \frac{\kappa\left(T^{\prime}\right) d T^{\prime}}{\left[\left(\kappa\left(T^{\prime}\right) \frac{d T}{d z}\right)_{T_{t i p}}^{2}+\frac{4}{r_{E}} \int_{\tau=T^{\prime}}^{T_{t i p}} q_{E} \kappa d \tau-\frac{4}{r_{E}} \int_{\tau=T^{\prime}}^{T_{t i p}} \epsilon_{t o t} \sigma_{S B} \tau^{4} \kappa d \tau-2 \int_{\tau=T^{\prime}}^{T_{t i p}} \frac{\kappa^{2}}{\lambda_{p e n}^{2}}\left(\frac{\partial \tau}{\partial \varphi}\right)_{\varphi_{e}} d \tau\right]^{1 / 2}}(4)
$$

$z=0$ is the position of the end face of the electrode, the integration constants are $T(z=0)=T_{t i p}$ and $(\kappa d T / d z)_{T_{t i p}}$.

According to [28] the first and second term within the square root under the fraction bar on the right hand side of eq. 4 can be written as:

$$
\left(\kappa \frac{d T}{d z}\right)_{T_{t i p}}^{2}+\frac{4}{r_{E}} \int_{\tau=T}^{T_{t i p}} q_{E}(\tau) \kappa d \tau=\frac{\left(P_{\text {in }}-P_{r}\right)^{2}-P_{c}^{2}}{\left(\pi r_{E}^{2}\right)^{2}}
$$

$P_{i n}$ is the total power input into the electrode and $P_{r}$ the radiant power, which is emitted from the end face of the electrode. The quantity

$$
P_{c}=2 \pi r_{E} \int_{0}^{z} q_{E} d z, \quad \text { with } q_{E} \neq 0 \text { for } z<r_{E}
$$

represents the power input into the cylinder barrel of the rod, which can be neglected as was shown in [28] if the major part of the power is supplied into the end face of the electrode. For $P_{c}^{2} /\left(P_{i n}-P_{r}\right)^{2}<<1$ the temperature distribution along the electrode can be written as:

$$
z(T) \approx \int_{T^{\prime}=T}^{T_{t i p}} \frac{\kappa\left(T^{\prime}\right) d T^{\prime}}{\left[\left(\frac{P_{i n}-P_{r}}{\pi r_{E}^{2}}\right)^{2}-\frac{4}{r_{E}} \int_{\tau=T^{\prime}}^{T_{t i p}} \epsilon_{t o t} \sigma_{S B} \tau^{4} \kappa d \tau-2 \int_{\tau=T^{\prime}}^{T_{t i p}} \frac{\kappa^{2}}{\lambda_{p e n}^{2}}\left(\frac{\partial \tau}{\partial \varphi}\right)_{\varphi_{e}} d \tau\right]^{1 / 2}}(7)
$$

The integral can be used to simulate a temperature distribution $z_{\text {sim }}(T)$ if measured values for $(\partial T / \partial \varphi)_{\varphi_{e}}$ and estimated values for $T_{t i p}$ and $P_{i n}-P_{r}$ are inserted.

Measured values $z_{\text {meas }}(T)$ between a lower temperature $T_{l}$ and an upper temperature $T_{u p}$ will be used to determine appropriate values of $T_{t i p}$ and $P_{i n}$ by searching the minimum of

$$
f\left(P_{\text {in }}, T_{\text {tip }}\right)=\int_{T_{l}}^{T_{u p}}\left[z_{\text {meas }}-z_{\text {sim }}\right]^{2} d T .
$$

Compared to [6] the new procedure offers the possibility to take into account the variation $(\partial T / \partial \varphi)_{\varphi_{e}}$ in the vicinity of the electrode tip. But the fundamental problem with the exaggerated temperature at the electrode tip and the limited penetration depth $\lambda_{\text {pen }}$ is not really removed. On the other hand the amplitude modulation of $T_{t i p}(\varphi)$ in dependence on the phase modulation of the power input $P_{i n}(\varphi)$ :

$$
\triangle T_{\text {tip }}(\varphi) \propto \frac{\triangle P_{i n}(\varphi)}{\rho_{M} c_{p} \pi r_{E}^{2} \lambda_{p e n} 2 \pi f}
$$


decreases as $\lambda_{\text {pen }}$ with $f^{-1 / 2}$, where $\pi r_{E}^{2} \lambda_{\text {pen }}$ represents the volume at the electrode tip in which a temperature variation takes place. It mitigates the problem with the modulation of $T_{t i p}$ with increasing frequency.

In [28] it was shown that the phase resolved experimental determination of $T_{\text {tip }}$ is afflicted with an error on the order of $\triangle T_{\text {tip }} \approx 50 \mathrm{~K}$ and $P_{\text {in }}$ with an error on the order of $10 \%$. Errors on this order of magnitude have to be taken into account appraising the measurements of $T_{\text {tip }}(\varphi)$ and $P_{i n}(\varphi)$ given below.

\section{Spectroscopic particle density measurement}

Phase resolved measurements of the $D y$ atom and ion density are carried out by the same measuring procedure as in [16]. Only the operating frequencies are extended up to $2 \mathrm{kHz}$. In addition the lamp filling is modified in part. Different to the pyrometric measurements the image of the vertically operated lamp is rotated through $90^{\circ}$ by the Dove prism around the axis of vision so that it is oriented perpendicular to the slit of the spectrograph. The slit captures the emission of a discharge cross section at a distance of $125 \mu \mathrm{m}$ from the electrode tip.

A LTE plasma with an equal temperature for all species, which represents the local plasma temperature $T$, and a Boltzmann-population of excited states is assumed by reason of the high filling gas pressure. The assumption was confirmed recently by a combination of absorption and emission spectroscopy at YAG lamps [11, 32].

In a first step the plasma temperature is determined using the emission of two overlapping mercury lines [33]. At first the absolute intensity of the two lines at $\lambda_{1}=576.96 \mathrm{~nm}$ and $\lambda_{2}=579.07 \mathrm{~nm}$ of atomic mercury is measured in dependence on the distance from the discharge axis. This results in a more or less axial symmetric distribution of the radiance of the two lines being integrated over a wavelength interval, covering the two lines, calibrated in absolute units. The sum of the emission coefficients of the two lines $\epsilon_{u l}=\epsilon_{1}+\epsilon_{2}$ is determined in dependence on the distance $r$ from the discharge axis by an inverse Abel transformation. The summed up emission coefficients $\epsilon_{u l}$ are related to the plasma temperature according to:

$$
\epsilon_{u l}=\frac{h c_{0}}{4 \pi} \frac{p_{0}}{k_{B} T} \frac{1}{Q_{H g}(T)}\left[\frac{g_{1} A_{1 l}}{\lambda_{1}} \exp \left(-\frac{E_{1}}{k_{B} T}\right)+\frac{g_{2} A_{2 l}}{\lambda_{2}} \exp \left(-\frac{E_{2}}{k_{B} T}\right)\right]
$$

where $p_{0}$ is the pressure of the mercury vapour, $Q_{H g}(T)$ the partition function of $\mathrm{Hg}$ atoms, given by $Q_{H g}(T<7000 \mathrm{~K}) \approx 1, A_{1}$ and $A_{2}$ the transition probabilities of the two lines, $g_{1}$ and $g_{2}$ the statistical weights and $E_{1}$ and $E_{2}$ the excitation energies of the upper line levels. Numerical values of the line data needed in eq. 10 are given in table 2. The other symbols in eq. 10 have the usual meaning. Eq. 10 is used to determine the plasma temperature $T(r)$ from the measured results $\epsilon_{u l}(r)$. 
Investigating the influence of the operating frequency on the gas phase emitter effect of Dy11

\begin{tabular}{ll}
\hline$\lambda_{1}=576.96 \mathrm{~nm}$ & $\lambda_{2}=579.07 \mathrm{~nm}$ \\
\hline$g_{1} A_{1}=1.18 \cdot 10^{8} \mathrm{~s}^{-1}$ & $g_{2} A_{2}=1.07 \cdot 10^{8} \mathrm{~s}^{-1}$ \\
$E_{1}=8.849 \mathrm{eV}$ & $E_{2}=8.842 \mathrm{eV}$ \\
\hline
\end{tabular}

Table 2. Spectroscopic data for the calculation of mercury temperature from $\mathrm{Hg}$-line intensities

The density $N_{a}$ of the $D y$ atoms and $N_{i}$ of the $D y$ ions are obtained from the spectroscopically measured local emission coefficient $\epsilon_{u l}^{a}$ of an atomic line and $\epsilon_{u l}^{i}$ of an ionic line emitted by optical transitions between an upper level $u$ and a lower level $l$.

An appropriate atomic line is the $695.81 \mathrm{~nm}$ line, a resonance line which is nevertheless optically thin by reason of the low transition probability. Comparisons of line profiles in [16] and of plasma temperatures measured with mercury lines and with $D y$ lines, respectively, in [32] have confirmed that the $695.81 \mathrm{~nm}$ line is really optically thin. A suitable $D y$ ion line with sufficiently high intensity is the $394.46 \mathrm{~nm}$ line. The specific constants of the two lines are given in table 3 [34].

\begin{tabular}{l|l}
\hline$\lambda_{a}=695.81 \mathrm{~nm}$ & $\lambda_{i}=394.46 \mathrm{~nm}$ \\
\hline$g_{a}=15$ & $g_{i}=18$ \\
$A_{a}=8.766 \cdot 10^{4} \mathrm{~s}^{-1}$ & $A_{i}=2.550 \cdot 10^{7} \mathrm{~s}^{-1}$ \\
$E_{a}=1.7815 \mathrm{eV}$ & $E_{i}=3.1424 \mathrm{eV}$ \\
\hline
\end{tabular}

Table 3. Spectroscopic data for the calculation of dysprosium atom and ion densities from Dy-atom- and ion-line intensities

The emission coefficients $\epsilon_{u l}^{a}$ and $\epsilon_{u l}^{i}$ are the result of an inverse Abel transformation of the lateral radiance distribution of the atomic line at $\lambda_{a}=695.81 \mathrm{~nm}$ and of the ionic line at $\lambda_{i}=394.46 \mathrm{~nm}$, being determined in absolute units in front of the electrode. Both emission coefficients are related to the population densities of the upper line levels $N_{u}$ according to:

$$
\epsilon_{u l}=\frac{1}{4 \pi} N_{u} A_{u l} \frac{h c_{0}}{\lambda_{u l}} .
$$

The densities of the upper line levels $N_{u}$ are related to those of the total densities $N$ according to:

$$
N=N_{u} \frac{Q(T)}{g_{u}} \exp \left(\frac{E_{u}}{k_{B} T}\right)
$$

where $Q(T)$ is the partition function of the particle species being considered, $g_{u}$ the statistical weight and $E_{u}$ the excitation energy of the upper level. With eq. 11 the 
population density of the upper emitting atomic state $N_{a, u}$ and ionic state $N_{i, u}$ are calculated and in a second step with eq. 12 the total atom density $N_{a}$ and total ion density $N_{i}$.

The error margins of $N_{a}$ and $N_{i}$ may be estimated making use of the logarithmic derivative of eq. 12 :

$$
\left|\frac{\triangle N}{N}\right|=\left|\frac{\triangle N_{u}}{N_{u}}\right|+\left|\frac{E_{u}}{k_{B} T} \frac{\triangle T}{T}\right|+\left|\frac{\partial \ln Q}{\partial \ln T} \frac{\triangle T}{T}\right|
$$

The error margin of $N_{u}$ is composed of the error margin of $\epsilon_{u l}$ and that of $A_{u l}$ :

$$
\left|\frac{\triangle N}{N}\right|=\left|\frac{\triangle \epsilon_{u l}}{\epsilon_{u l}}\right|+\left|\frac{\triangle A_{u l}}{A_{u l}}\right|
$$

The emission coefficients $\epsilon_{u l}$ are afflicted at least with an error of $20 \%$. It is mainly caused by the inverse Abel transformation, which reacts, particularly on the axis, quite sensitively on deviations from symmetry of the lateral distribution of the line intensity. It has also to be taken into account that the transmission of the lamp tube may be reduced during lamp operation. Additionally the SNR especially of the $394.46 \mathrm{~nm}$ line becomes low, if for high operating frequencies short exposure times are required. Besides the knowledge of the transition probability $A_{u l}$ is correct not better than $10 \%$. Another important error source is according to eq. 13 the uncertainty of the plasma temperature $\triangle T / T$ by reason of the multiplier $E_{u} / k_{B} T$. The plasma temperature in front of the electrode is $T \approx 7000 \mathrm{~K}$ [16], with an error margin of about $4 \%$. The multiplier is $E_{a, u} / k_{B} T \approx 3$ for the atoms $N_{a}$ and $E_{i, u} / k_{B} T \approx 5$ for the ions $N_{i}$. The multiplier of the third term in eq. 13 is roughly $\partial \ln Q / \partial \ln T \approx 1$ so that its contribution to the total error margin is minor.

The resulting error margins are: $\left|\triangle N_{a} / N_{a}\right| \cdot 100 \geq 40 \%$ and $\left|\triangle N_{i} / N_{i}\right| \cdot 100 \geq$ $50 \%$. They reveal that the numerical values of the particle densities obtained by the spectroscopic measurements are only in the order of magnitude correct. But the variations in dependence on phase, $\partial N_{a} / \partial \varphi$ and $\partial N_{i} / \partial \varphi$, represent a quite reliable information. Subsequently the reported values of $T, N_{a}$ and $N_{i}$ are averages over a rotational symmetric cross section with the same diameter as the electrode.

\section{Results of measurements at the YAG lamp}

Results for the density of $D y$ atoms $N_{a}$ and $D y$ ions $N_{i}$, of the electrode tip temperature $T_{\text {tip }}$ and of the input power $P_{i n}$ into the electrode are presented in dependence on the phase angle $\varphi$. All pyrometric measurements are performed at the upper electrode, all spectroscopic measurements in front of the upper electrode. Measurements are executed at operating frequencies of 1, 10, 25, 50, 100, 250, $500 \mathrm{~Hz}, 1$ and $2 \mathrm{kHz}$. For frequencies up to $100 \mathrm{~Hz}$ measurements are carried out at 20 equidistant phase angles $\varphi_{\nu}=0.1 \pi \nu$, $(\nu=1-20)$ over one period. For $f>100 \mathrm{~Hz}$ the number of measuring points is individually adjusted to a lower rate so that the SNR can be kept sufficiently high for a 
suitable measurement. For $f=2 \mathrm{kHz}$ mostly only one measurement within the anodic and cathodic half period was possible. In the subsequent figures the measurements within the anodic half period are presented between $\varphi=0$ and $\varphi=\pi$, and those within the cathodic half period between $\varphi=\pi$ and $\varphi=2 \pi$.

\subsection{YAG lamp with $\mathrm{Hg}$ filling only}

At first the properties of a YAG lamp operated with a pure mercury vapour atmosphere are investigated in dependence on the operating frequency for comparison with lamps seeded with metal halides. The electrode diameter amounts to $d_{E}=0.36 \mathrm{~mm}$, its extension into the tube to $l_{E}=5 \mathrm{~mm}$. The lamp is operated with a switched dc current with an amplitude of $0.8 \mathrm{~A}$. For this current amplitude the heating of the electrode is higher within the anodic phase than within the cathodic phase at least for dc operation and for ac operation with low commutation frequencies. The formation of a spot mode at the beginning of the cathodic phase is avoided by this choice of parameters [24]. The operating frequencies are $f=1,10,25,50,100,250,500 \mathrm{~Hz}$ and $1 \mathrm{kHz}$.

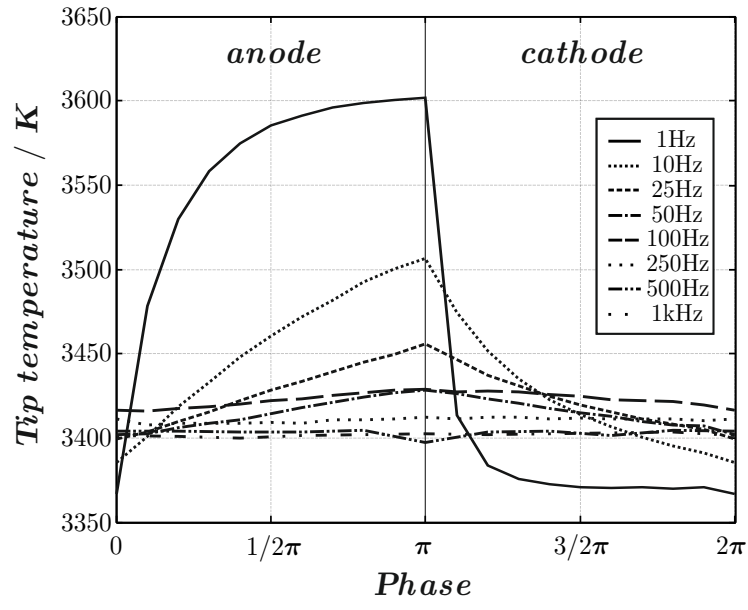

(a) Electrode tip temperature

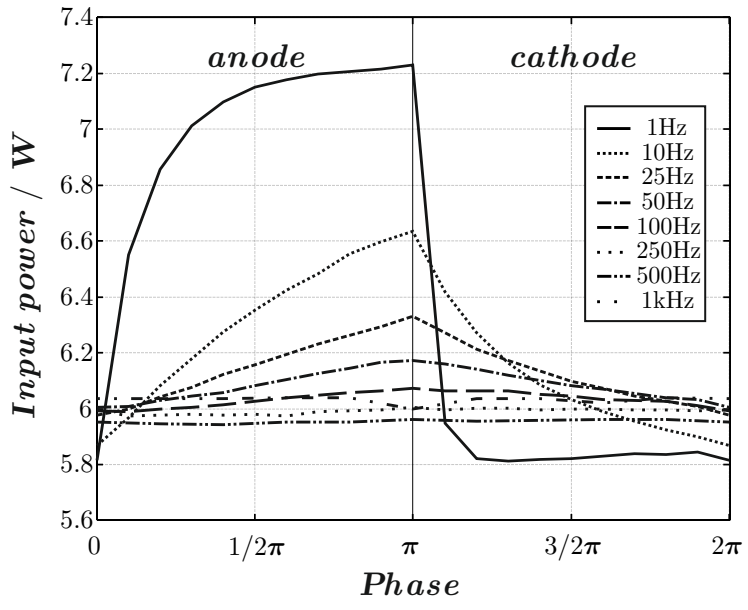

(b) Electrode input power

Figure 1. Electrode tip temperature $T_{\text {tip }}(\varphi)$ and input power $P_{\text {in }}(\varphi)$ over one period for various frequencies. Parameter: $H g$, electrode diameter $d_{E}=0.36 \mathrm{~mm}$, electrode length $l_{E}=5 \mathrm{~mm}, i=0.8 \mathrm{~A}$ switched-dc

Figure 1(a) shows the variation of the electrode tip temperature $T_{\text {tip }}(\varphi)$ in dependence on the phase angle $\varphi$ and figure 1(b) the variation of the power input $P_{\text {in }}(\varphi)$. Figure 1(a) and 1(b) demonstrate a pronounced reduction of $T_{t i p}(\varphi)$ and $P_{i n}(\varphi)$ within the anodic phase and a weak lifting of both quantities within the cathodic phase with increasing operating frequency. Small deviations from the general trend e.g. for $f=100 \mathrm{~Hz}$ are within the error bars. For $f \geq 250 \mathrm{~Hz}$ the differences between the anodic and cathodic phase become almost indistinguishable. The nearly constant magnitude of $T_{t i p}$ and $P_{i n}$ is only marginally higher than $T_{t i p}$ and $P_{i n}$ of a dc operated cathode, 
which is loaded with the same current amplitude. The results are in general agreement with those obtained by measurements at the model lamp with a pure argon filling [28].

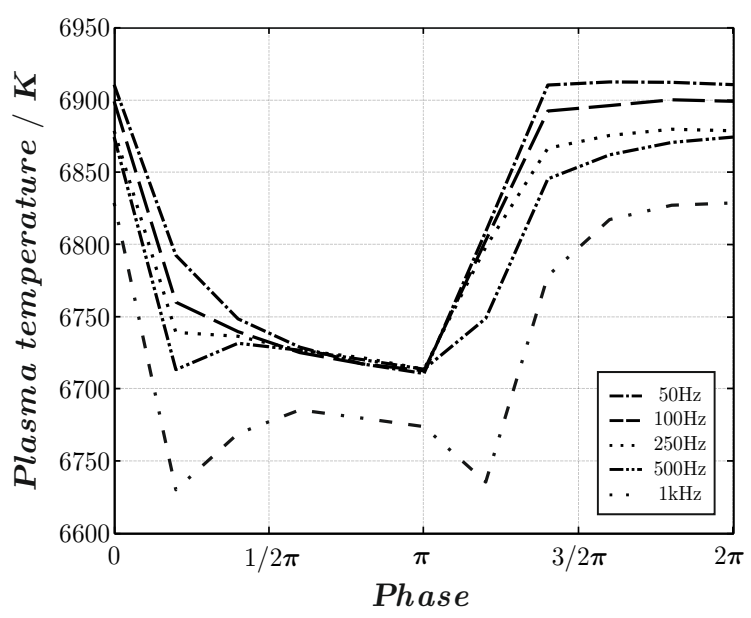

Figure 2. Plasma temperature $T(\varphi)$ over one period for various frequencies measured at a distance of $125 \mu \mathrm{m}$ in front of the electrode surface. Parameter: $\mathrm{Hg}$, electrode diameter $d_{E}=0.36 \mathrm{~mm}$, electrode length $l_{E}=5 \mathrm{~mm}, i=0.8 \mathrm{~A}$ switched-dc

In figure 2 the variation of the plasma temperature $T$ at a distance $z=125 \mu \mathrm{m}$ from the electrode tip within a YAG tube is given in dependence on the phase angle $\varphi$. The tube parameters are the same as in figure 1, the amplitude of the switched dc current is $i=0.8 \mathrm{~A}$, the operating frequencies are $f=50,100,250,500 \mathrm{~Hz}$ and $1 \mathrm{kHz}$. $T$ is deduced from the measured lateral distribution of the absolute intensities of the two mercury lines at $\lambda_{1}=576.96 \mathrm{~nm}$ and $\lambda_{2}=579.07 \mathrm{~nm}$. The plasma temperature $T$ is approximately $200 \mathrm{~K}$ higher within the cathodic phase than within the anodic phase. It reflects the generation of an ion current in front of the cathode, to balance the power consumption by thermionic electron emission of the electrode within the cathodic phase. It decreases with increasing operating frequency namely approximately by $100 \mathrm{~K}$ within the cathodic phase and by $50 \mathrm{~K}$ within the anodic phase.

\subsection{YAG lamp seeded with $D y I_{3}$}

In a next step YAG lamps with the same design as the mercury lamp but additionally seeded with $1 \mathrm{mg} D y I_{3}$ are investigated. In particular the dimension of the electrodes is the same: $d_{E}=0.36 \mathrm{~mm}, l_{E}=5 \mathrm{~mm}$. Results for $N_{a}(\varphi), N_{i}(\varphi), T_{t i p}(\varphi)$ and $P_{i n}(\varphi)$ are given for a lamp which is operated with a switched dc current of $i=0.8 \mathrm{~A}$. The operating frequencies are $f=25,50,100 \mathrm{~Hz}$ and $2 \mathrm{kHz}$.

Figure 3(a) and 3(b) show the $D y$ atom density $N_{a}(\varphi)$ and the $D y$ ion density $N_{i}(\varphi)$, respectively, both versus phase angle $\varphi$. The $D y$ atom density grows for frequencies $f \leq 50 \mathrm{~Hz}$ within the cathodic phase up to $N_{a}=10^{22} \mathrm{~m}^{-3}$, which is an order 
Investigating the influence of the operating frequency on the gas phase emitter effect of Dy 15

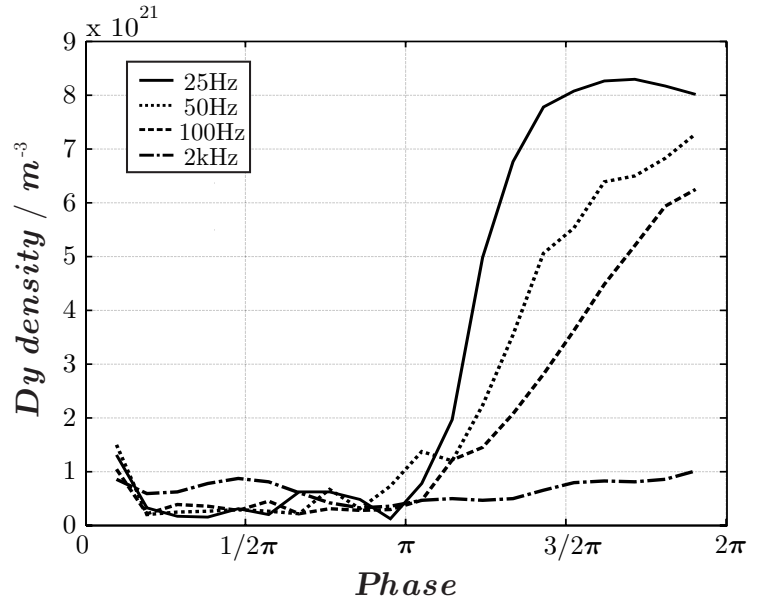

(a) Atoms

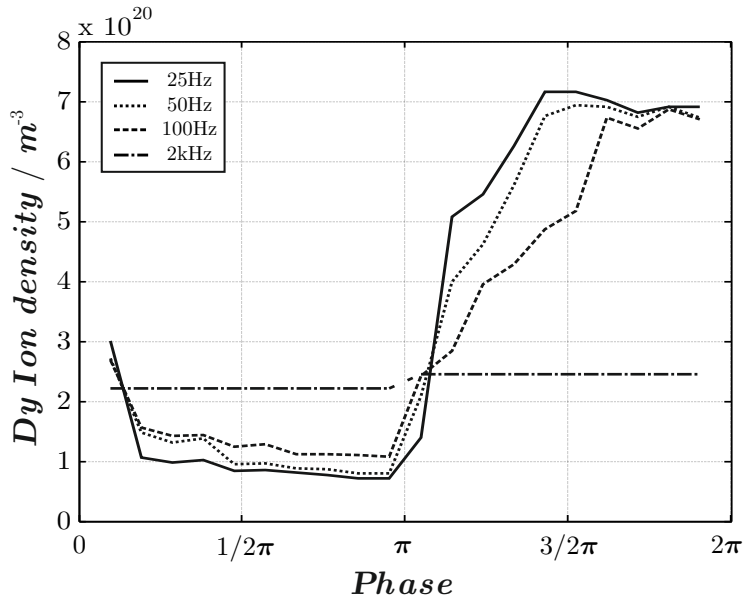

(b) Ions

Figure 3. Dysprosium atom density $N_{a}(\varphi)$ and ion density $N_{i}(\varphi)$ over one period for various frequencies at a distance of $125 \mu \mathrm{m}$ in front of the electrode. Parameter: $D y I_{3}$, electrode diameter $d_{E}=0.36 \mathrm{~mm}$, electrode length $l_{E}=5 \mathrm{~mm}, i=0.8 \mathrm{~A}$ switched-dc

of magnitude higher than within the anodic phase. A saturation of $N_{a}$ occurs approximately after $\tau_{a} \approx 10 \mathrm{~ms}$. For $f>50 \mathrm{~Hz}$ the half period is too short to achieve the saturated $D y$ atom density. As a consequence the maximum value of $N_{a}$ drops down within the cathodic phase with increasing operating frequency. The $D y$ ion density $N_{i}$ rises as well from a value of approximately $10^{20} \mathrm{~m}^{-3}$ within the anodic phase to $7 \cdot 10^{20} \mathrm{~m}^{-3}$ within the cathodic phase. A saturation of $N_{i}$ is already achieved within $\tau_{i} \approx 5 \mathrm{~ms}$. Therefore the half period becomes too short for $f>100 \mathrm{~Hz}$ to sustain the modulation of $N_{i}$ being observed at lower operating frequencies. For $f=2 \mathrm{kHz}$ the ion densities within the anodic and cathodic phase have matched by a lifting of $N_{i}$ within the anodic phase and a lowering of $N_{i}$ within the cathodic phase.

In figure 4(a) values of $N_{a}$ within the anodic and cathodic phase averaged over the half period are given in dependence on the operating frequency $f$ and in figure 4(b) the corresponding values of $N_{i}(f) . N_{a}$ is nearly independent of $f$ within the anodic phase. But within the cathodic phase it decreases monotonically by an order of magnitude from a high value at low operating frequencies down to the value of $N_{a}$ in front of the anode at higher frequencies. A quite similar behaviour is observed for the $D y$ ion density $N_{i}$. However, different to $N_{a}$, the ion density $N_{i}$ in front of the anode increases at higher frequencies by a factor of approximately two.

The effects of $D y I_{3}$ on the electrode tip temperature $T_{t i p}(\varphi)$ and on the power input $P_{\text {in }}(\varphi)$ are presented in figure 5(a) and in figure 5(b), respectively. A comparison of $T_{t i p}(\varphi)$ in figure 1(a) and figure 5(a) clearly demonstrates a reduction of $T_{t i p}(\varphi)$ by approximately $400 \mathrm{~K}$ for all operating frequencies as a result of the addition of $D y I_{3}$. The reduction of $T_{t i p}(\varphi)$ with increasing operating frequency not only within the anodic 
Investigating the influence of the operating frequency on the gas phase emitter effect of Dy 16

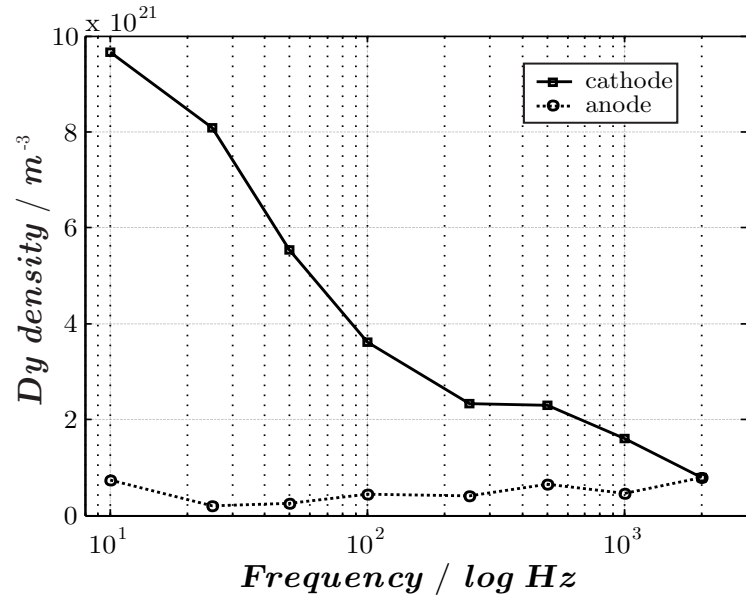

(a) Atoms

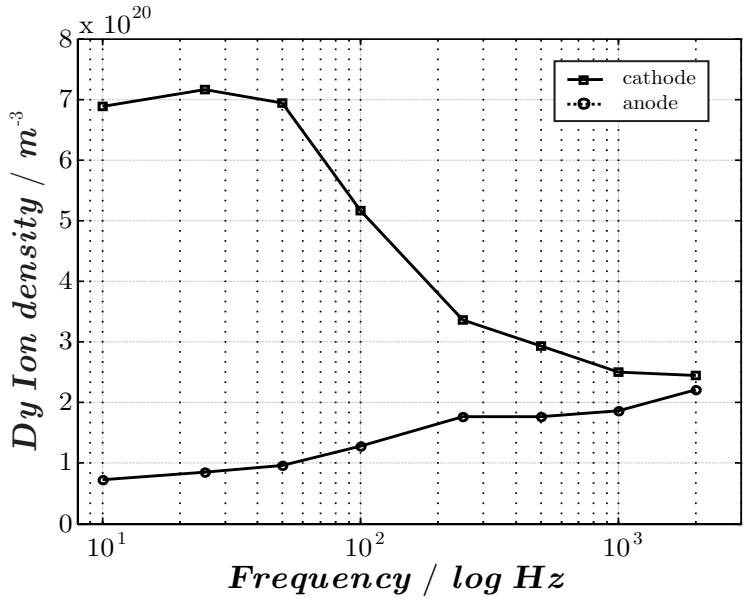

(b) Ions

Figure 4. Maximum values of the $D y$ atom density $N_{a}(f)$ and $D y$ ion density $N_{i}(f)$ depending on frequency at a distance of $125 \mu \mathrm{m}$ in front of the electrode. Parameter: $D y I_{3}$, electrode diameter $d_{E}=0.36 \mathrm{~mm}$, electrode length $l_{E}=5 \mathrm{~mm}, i=0.8 \mathrm{~A}$ switched-dc

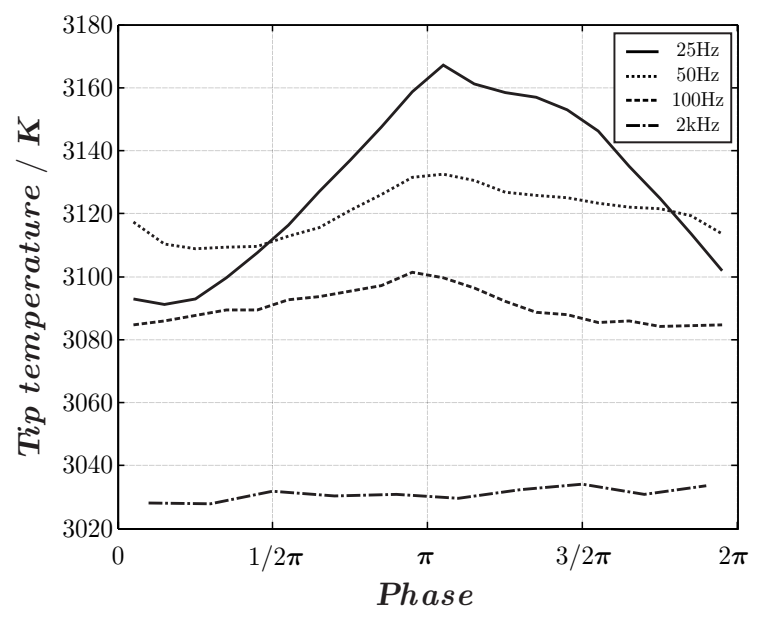

(a) Electrode tip temperature

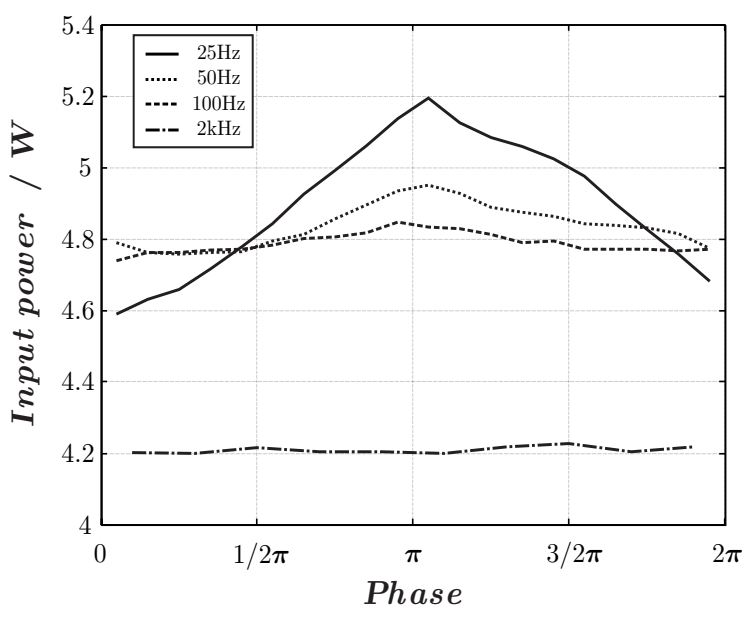

(b) Electrode input power

Figure 5. Electrode tip temperature $T_{\text {tip }}(\varphi)$ and input power $P_{\text {in }}(\varphi)$ over one period for various frequencies. Parameter: $D y I_{3}$, electrode diameter $d_{E}=0.36 \mathrm{~mm}$, electrode length $l_{E}=5 \mathrm{~mm}, i=0.8 \mathrm{~A}$ switched-dc

phase but also within the cathodic phase is particularly noteworthy. This reduction within the cathodic phase differs from the weak increase observed in a pure argon or mercury atmosphere (figure 1(a)). Quite similar statements can be made if $P_{\text {in }}(\varphi)$ in figure 1(b) and figure 5(b) are compared. At $f=2 \mathrm{kHz}$ the phase averaged power input $P_{i n}$ is reduced by approximately $30 \%$ compared to a lamp with a pure mercury filling.

Complementary the effect of different electrode diameters is investigated. For this 
Investigating the influence of the operating frequency on the gas phase emitter effect of Dy17

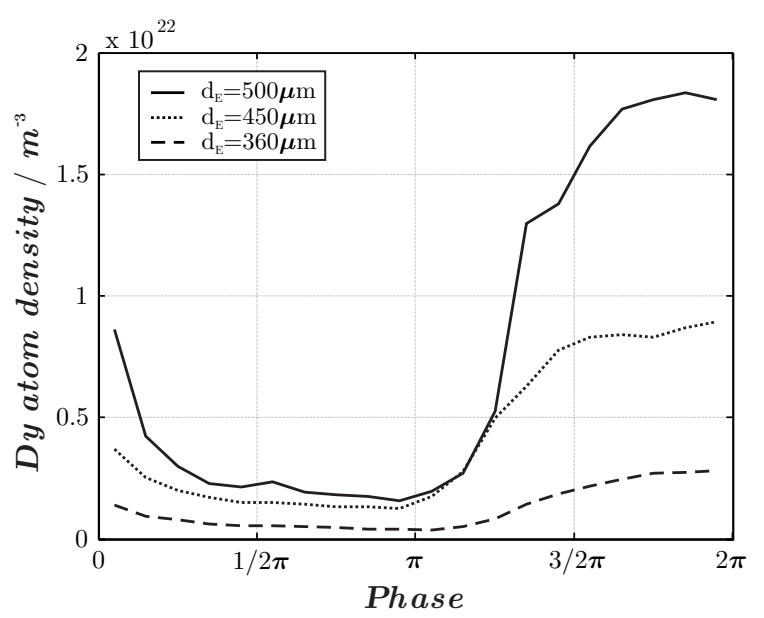

(a) Dy atom density, $f=50 \mathrm{~Hz}$

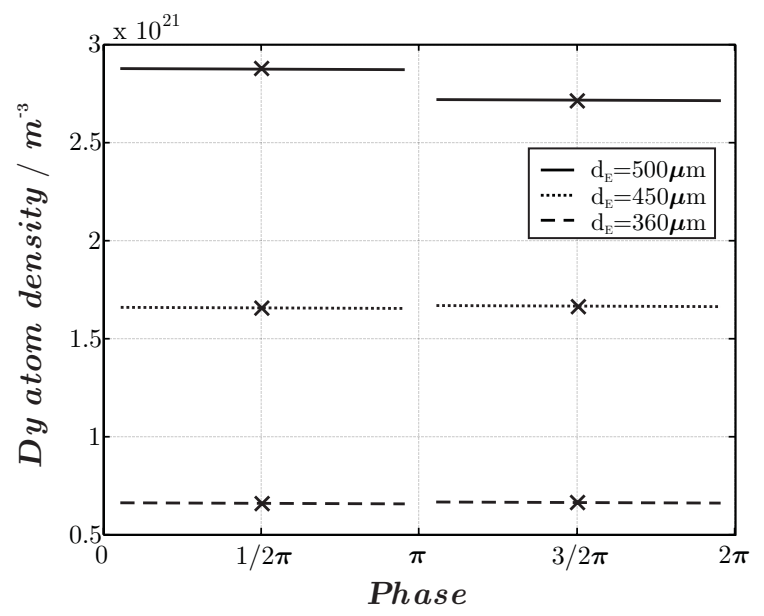

(c) $D y$ atom density, $f=2 \mathrm{kHz}$

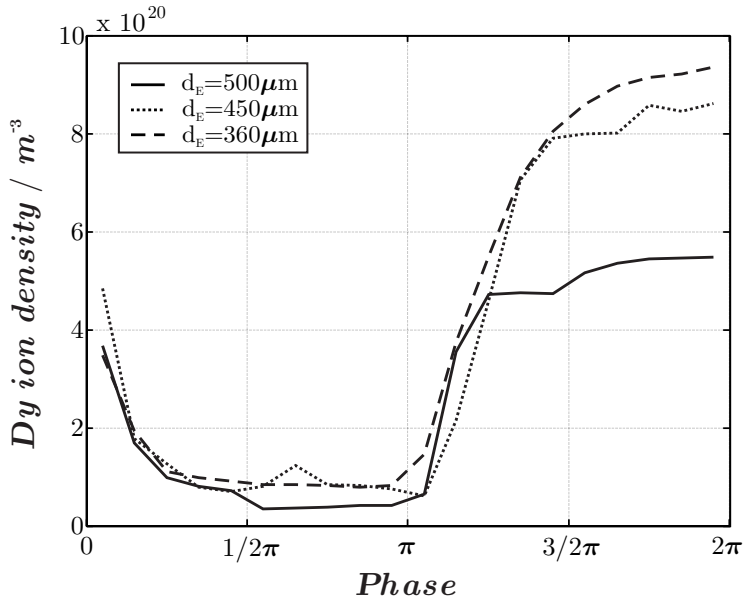

(b) $D y$ ion density, $f=50 \mathrm{~Hz}$

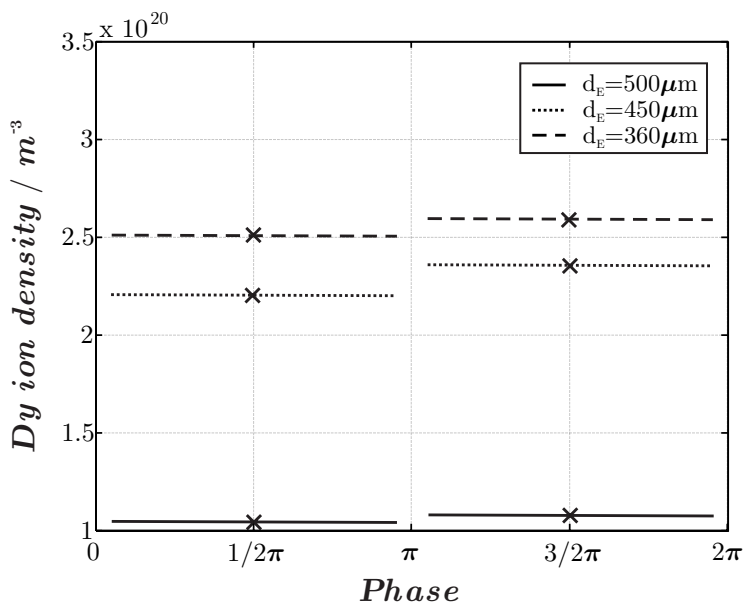

(d) $D y$ ion density, $f=2 \mathrm{kHz}$

Figure 6. Dy atom density $N_{a}(\varphi)$ and ion density $N_{i}(\varphi)$ for frequencies of $50 \mathrm{~Hz}$ and $2 \mathrm{kHz}$ over one period at a distance of $125 \mu \mathrm{m}$. Parameter: $D y I_{3}$, electrode diameter $d_{E}=0.36,0.45$ and $0.5 \mathrm{~mm}$, electrode length $l_{E}=5 \mathrm{~mm}, i=0.8 \mathrm{~A}$ switched-dc

purpose spectroscopic and pyrometric measurements are compared, being accomplished on lamps, which differ only by the electrode diameter. The different diameters are: $d_{E}=360,450$ and $500 \mu \mathrm{m}$. The lamps are operated with a switched dc current of $i=0.8 \mathrm{~A}$ at operating frequencies of $f=50 \mathrm{~Hz}$ and $2 \mathrm{kHz}$. In figure 6 measurements of the densities of $D y$ atoms and $D y$ ions in front of the upper electrode are given. Figures 6(a) and 6(b) show $N_{a}(\varphi)$ and $N_{i}(\varphi)$, respectively, at $f=50 \mathrm{~Hz}$ for all three diameters. Within the cathodic phase distinctly higher densities of both $D y$ atoms and ions are found than within the anodic phase for all three electrode diameters. In the cathodic phase the density $N_{a}$ becomes larger, $N_{i}$ smaller with increasing electrode diameter. It corresponds to a decrease of the electron temperature within the boundary layer in front of the cathode with increasing electrode diameter at constant current according to [3], figure 6. Figure 6(c) and figure 6(d) represent only average values of $N_{a}$ and 
$N_{i}$ within the anodic and cathodic half period for $f=2 \mathrm{kHz}$. For this high frequency the tolerable exposure time was too short and therefore the recorded number of counts too small for a more detailed phase resolution. Not only for $d_{E}=360 \mu \mathrm{m}$ but also for $d_{E}=450,500 \mu \mathrm{m}$ a reduction of $N_{a}$ within the cathodic phase towards the lower value within the anodic phase is observed. Also the ion densities $N_{i}$ in both phases match at $2 \mathrm{kHz}$ due to a lifting of $N_{i}$ within the anodic phase and a lowering within the cathodic phase.

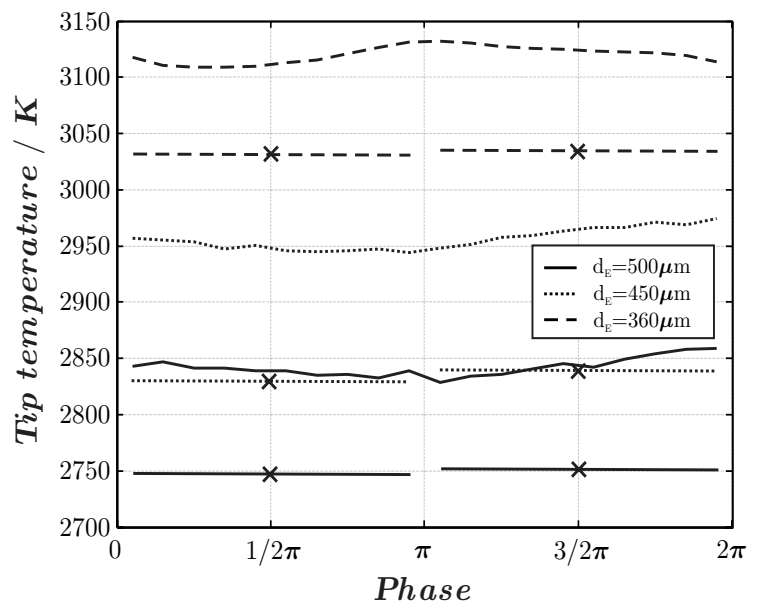

(a) Electrode tip temperature

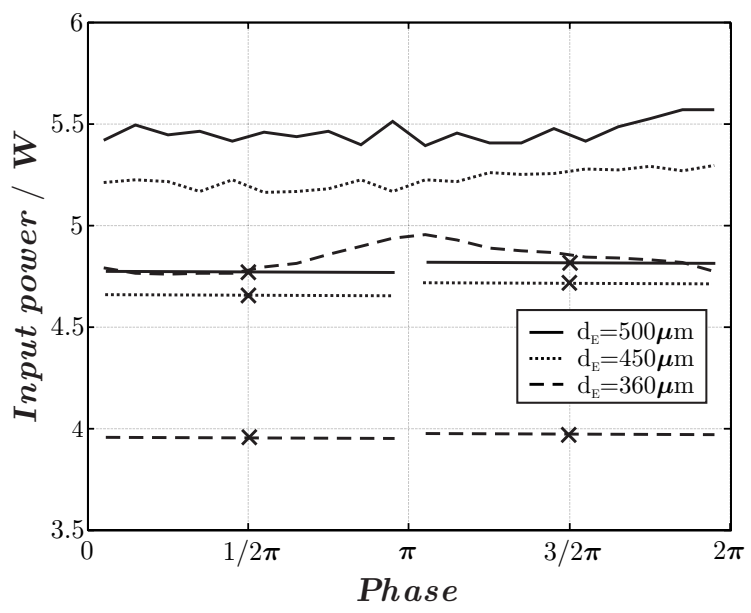

(b) Electrode input power

Figure 7. Electrode tip temperature $T_{t i p}(\varphi)$ and input power $P_{i n}(\varphi)$ for frequencies of $50 \mathrm{~Hz}$ (curves) and $2 \mathrm{kHz}$ (crosses) over one period. Parameter: $D y I_{3}$, electrode diameter $d_{E}=0.36,0.45$ and $0.5 \mathrm{~mm}$, electrode length $l_{E}=5 \mathrm{~mm}, i=0.8 \mathrm{~A}$ switched$\mathrm{dc}$

Comparing the particle densities in figure 3 and figure 6 for an electrode diameter $d_{E}=360 \mu \mathrm{m}$ it has to be taken into account that $N_{a}(\varphi)$ and $N_{i}(\varphi)$ are measured in different lamps of the same type. The differences are on the same order of magnitude as the measuring errors of $N_{a}(\varphi)$ and $N_{i}(\varphi)$ given in section 4 .

In figure $7 T_{t i p}$ and $P_{i n}$ are compared for the three electrode diameters. $T_{t i p}$ is lowered by an increase of $d_{E}$, the opposite applies for $P_{i n}$. Both quantities are reduced by approximately the same amount for all three diameters, if the operating frequency is increased from $50 \mathrm{~Hz}$ to $2 \mathrm{kHz}$. The differences between $T_{t i p}$ and $P_{i n}$ for $d_{E}=360 \mu \mathrm{m}$ in figure 5 and figure 7 are negligible.

\subsection{YAG lamp seeded with $T l I$ and $D y I_{3}$}

Previous measurements have suggested [16] that the emitter effect of dysprosium may be perturbed by other ingredients within the MH lamp. To substantiate this suspicion a YAG lamp was investigated, which was seeded with $1.242 \mathrm{mg} D y I_{3}$ and $0.758 \mathrm{mg} \mathrm{TlI}$ 
Investigating the influence of the operating frequency on the gas phase emitter effect of Dy19

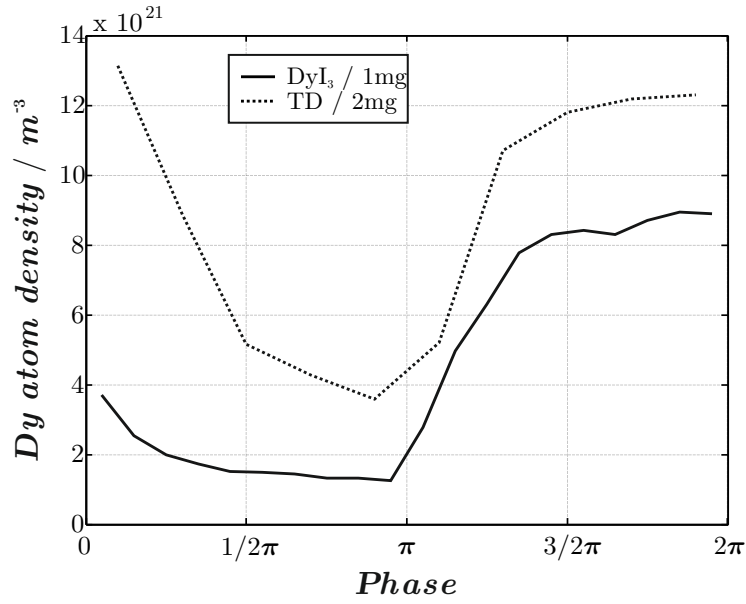

(a) $50 \mathrm{~Hz}, D y$ atom

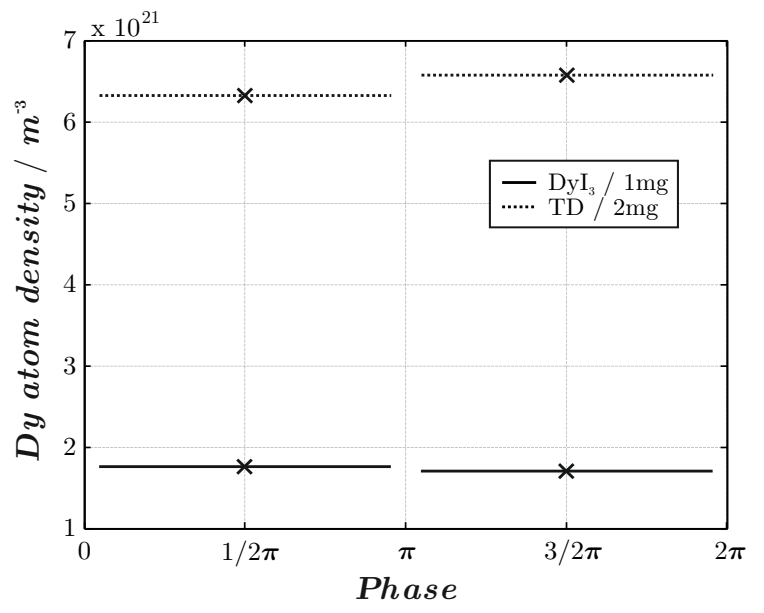

(c) $2 \mathrm{kHz}, D y$ atom

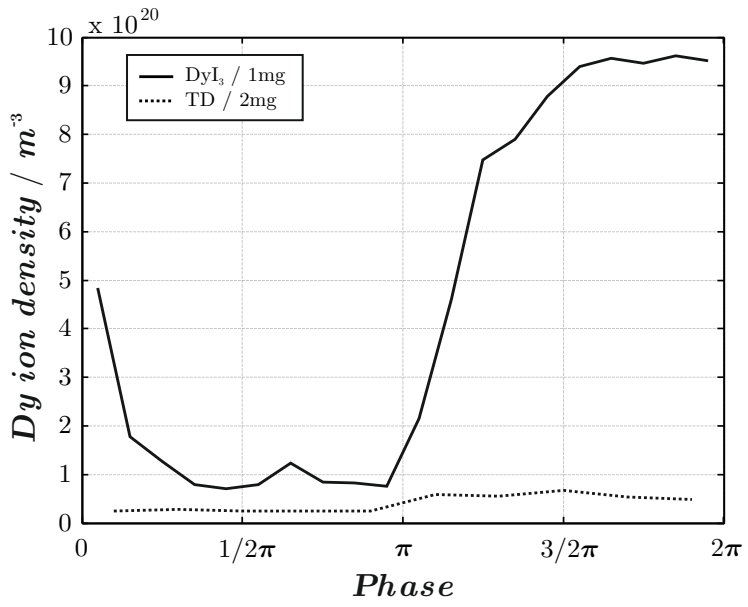

(b) $50 \mathrm{~Hz}, D y$ ion

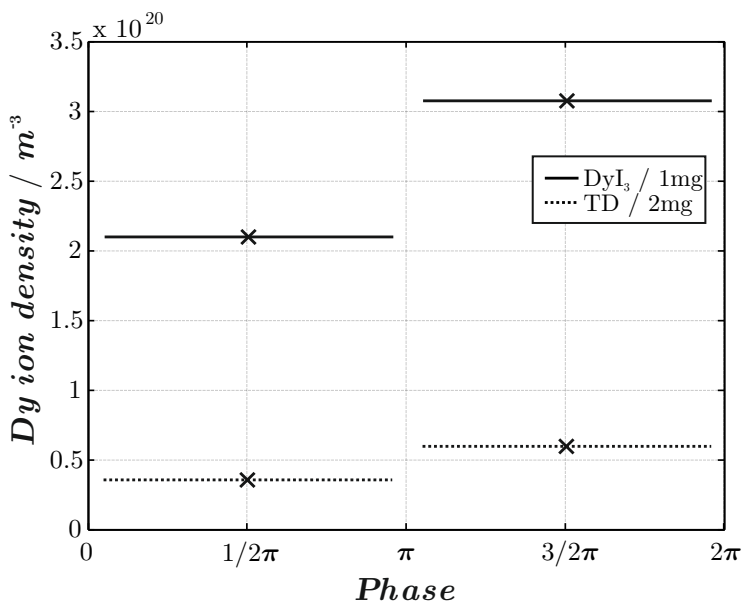

(d) $2 \mathrm{kHz}, D y$ ion

Figure 8. Phase resolved $D y$ atom density $N_{a}(\varphi)$ and ion density $N_{i}(\varphi)$ for a low and a high frequency $(50 \mathrm{~Hz}$ and $2 \mathrm{kHz})$ for different lamp fillings. For the high frequency the values are average values over the anodic respectively cathodic phase. Parameter: $D y I_{3}$, TD, electrode diameter $d_{E}=0.45 \mathrm{~mm}$, electrode length $l_{E}=5 \mathrm{~mm}, i=0.8 \mathrm{~A}$ switched-dc

(2 mg salt: $50 \mathrm{~mol} \% \mathrm{Dy} I_{3}$ and $50 \mathrm{~mol} \% \mathrm{TlI}$, as mentioned in table 1). The results which were obtained with this so called TD-lamp are compared with those, which were obtained with the so called D-lamp. It was seeded as the lamps being investigated in section 5.2 with $1 \mathrm{mg} D y I_{3}$. The electrode diameter was $d_{E}=450 \mu \mathrm{m}$. The lamps were operated with a switched dc current with an amplitude of $i=0.8 \mathrm{~A}$ being modulated with $f=50 \mathrm{~Hz}$ or $f=2 \mathrm{kHz}$. In figure 8 densities of $D y$ atoms and $D y$ ions are compared, which were measured in front of the upper electrode of the TD-lamp and D-lamp. Figure 8(a) and 8(b) represent the $D y$ atom density $N_{a}(\varphi)$ and the $D y$ ion density $N_{i}(\varphi)$, respectively, taken at an operating frequency of $50 \mathrm{~Hz}$ for the two lamps. For both lamps the $D y$ atom density $N_{a}$ within the cathodic phase is much higher than within the anodic phase. It is even higher within the TD-lamp than within the D-lamp 
corresponding to the higher amount of $D y I_{3}$ within the TD-lamp. The $D y$ ion density $N_{i}$ in the TD-lamp is only marginally higher within the cathodic phase than within the anodic phase, in contrast to the D-lamp. Figure 8(c) and figure $8(\mathrm{~d})$ give for $f=2 \mathrm{kHz}$ only average values of $N_{a}$ and $N_{i}$ within the anodic and cathodic half period by the same reason as in figure 6(c) and 6(d). The density of the $D y$ atoms $N_{a}$ within the cathodic phase is reduced to the level within the anodic phase while $N_{a}$ within the anodic phase keeps the same value with increasing frequency. As in figure $8(\mathrm{a}), N_{a}$ is higher within the TD-lamp than within the D-lamp. The density of the $D y$ ions $N_{i}$ within the TD-lamp remains on the same low level as in figure 8(b). The variation of $N_{i}$ within the D-lamp with increasing operating freqency was already discussed in section 5.2. In figure 9 courses of $T_{\text {tip }}(\varphi)$ measured within the TD-lamp and D-lamp are compared for the same operating conditions at $f=50 \mathrm{~Hz}$ and $f=2 \mathrm{kHz}$. Figure 9 clearly demonstrates that $T_{\text {tip }}$ is nearly $300 \mathrm{~K}$ lower within the D-lamp than within the TD-lamp.

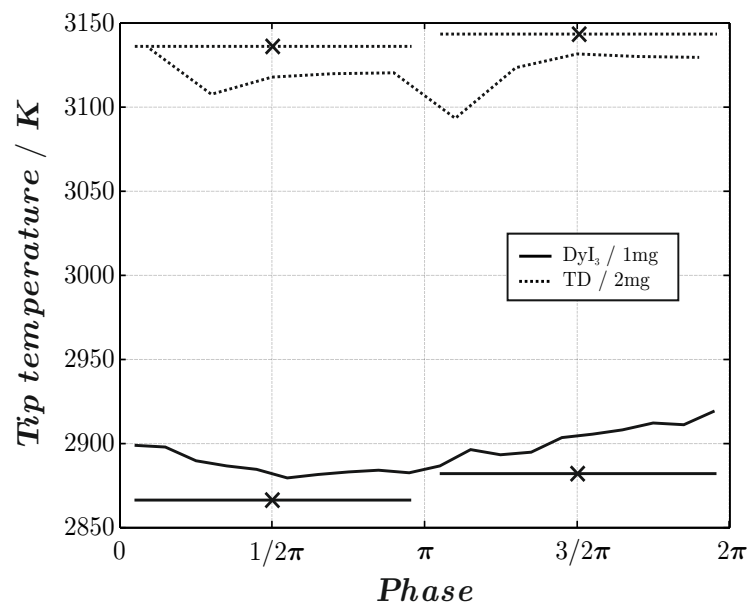

Figure 9. Phase resolved electrode tip temperature $T_{t i p}(\varphi)$ for a low and a high frequency $(50 \mathrm{~Hz}$ and $2 \mathrm{kHz})$ for different lamp fillings. For the high frequency the values are average values over the anodic and cathodic phase, respectively. Parameter: $D y I_{3}$, TD, electrode diameter $d_{E}=0.45 \mathrm{~mm}$, electrode length $l_{E}=5 \mathrm{~mm}, i=0.8 \mathrm{~A}$ switched-dc

\section{Interpretation of the measuring results}

The particle density and electrode temperature measurements reveal a strong correlation between the $D y$ ion density in front of the electrode, and the electrode tip temperature and power input into the electrode. Compared to an equivalent pure mercury lamp operated with the same current $i(t)$ they are significantly lowered, if a high dysprosium ion density is measured in front of the electrode, as is demonstrated in figure 1, figure 3 and figure 5. A high Dy ion density is succeeded, with some time delay, by a high $D y$ atom density, as is illustrated in figure 3 and figure 6, but a high $D y$ atom density 
is not accompanied in general by a high $D y$ ion density. An example of an exception is shown in figure 8 . The $D y$ ion and atom density is generally higher within the cathodic half period than within the anodic half period independently of the operating frequency (figure 3), the electrode diameter (figure 6) and the composition of the lamp filling (figure 8). But with increasing operating frequency a decline of the $D y$ ion and atom density within the cathodic phase is observed together with a distinct increase of the $D y$ ion density within the anodic phase as is demonstrated in figure 4. Moreover a delayed adjustment of the $D y$ density on the electrode polarity can be observed especially at the beginning of the anodic phase, as shown in figure 6(a) and figure 6(b).

The accumulation of $D y$ ions and, with a time delay of several milliseconds, of Dy atoms in front of the cathode can only be explained by a cataphoretic transport of $D y$ ions from the bulk plasma to the electrode region within the cathodic phase. The related reduction of the electrode tip temperature has to be attributed to a lowering of the work function $\Phi$ of the cathode. It reduces the power loss of the cathode via thermionic electron emission, and with it the power transfer needed from the plasma to the cathode. The lowering of $\Phi$ is the effect of a $D y$ monolayer on the electrode surface. The growth and destruction of the monolayer depends on the adsorption/desorption energy of the $D y$ atoms on the tungsten surface, on the electrode tip temperature and the replacement rate of the desorbed $D y$ atoms from the gas phase. The measuring results are a strong indication that a $D y$ ion current plays a decisive role for the formation of a $D y$ monolayer within a $\mathrm{MH}$ lamp.

An emitter effect generated by an ion current should be limited to the cathode. Therefore, the reduction of the electrode temperature also within the anodic phase for operating frequencies on the order of $1 \mathrm{kHz}$ has to be explained by a finite lifetime of the $D y$-monolayer. If it is on the order of $1 \mathrm{~ms}$, it will also reduce the work function $\Phi$ within the anodic phase for $f \geq 1 \mathrm{kHz}$. The heating of the electrode within the anodic phase is approximately given by:

$$
P_{i n} \approx i\left(\Phi+\frac{5}{2} \frac{k_{B} T_{e, a r c}}{e}\right),
$$

where $T_{e, a r c}$ is the average temperature of the electrons, which enter the constriction zone in front of the anode from the side of the bulk plasma. Therefore, a reduction of $\Phi$ diminishes the power input into the electrode not only within the cathodic phase but also within the anodic phase. It will explain the distinct reduction of $T_{\text {tip }}$ at higher operating frequencies independently of the electrode diameter (figure 7).

The validity of eq. 15 at least for hot tungsten anodes is confirmed by measurements at the model lamp being analyzed in [10], section 5.2. The missing reduction of the power input into HID anodes operated with a dc current by doping them with $\mathrm{ThO}_{2}[9,10]$ does not provide an argument against the validity of eq. 15. Instead, it is 
an indication that an emitter effect is generated by a thorium ion current towards the cathode, also in case of thoriated tungsten electrodes for HID lamps [35]. A publication of detailed investigations of the emitter effect at thoriated tungsten electrodes for HID lamps is under way. Eq. 9 in [36] and eq. 15, as well as the electron temperatures in these equations, are different since they apply to different situations. Eq. 9 in [36] is a theoretical expression for the power flux density from the plasma sheath immediately in front of the electrode while eq. 15 describes the power transfer from the anodic boundary layer composed of the plasma sheath and the constriction zone to the anode.

The effect of an additional seeding of the YAG lamp with TlI is demonstrated in figure 8 and figure 9. The thallium additive inhibits the formation of $D y$ ions in front of the cathode as is shown by the measuring results in figure 8. The ionisation energy of $T l, E_{i}=6.11 \mathrm{eV}$, exceeds that of $D y$ only by $0.19 \mathrm{eV}$ so that the ionisation degree of $T l$ may be comparable with that of $D y$. But the vapour pressure of $T l$ is distinctly higher than that of $D y$. Assuming a cold spot temperature $T_{c s p}$ of the YAG lamp in the $1150-1200 \mathrm{k}$ range the corresponding pressure ratio $p(T l) / p(D y)$ amounts to 350 and 90, respectively [37]. The low values of the $D y$ ion density $N_{i}(\varphi)$ are reflected by a high electrode tip temperature $T_{t i p}$ in figure 9. Further investigations into the TD-lamp are needed to clarify the reduction of the $D y$ ion density but not of the $D y$ atom density in front of the cathode. An interpretation of the result is difficult, since the $D y$ atoms are accumulated by the cataphoretic transport of $D y$ ions in front of the cathode.

Another very common ingredient of $\mathrm{MH}$ lamps is sodium. Measuring results given in [16] raise suspicion that $N a$ ions displace $D y$ ions in front of the cathode so that the formation of a $D y$ monolayer on the electrode surface is hampered. The effect of $N a$ and of a salt mixture consisting of $\mathrm{NaI}, \mathrm{TlI}$ and $\mathrm{Dy} I_{3}$ on the electrode properties in MH lamps will be investigated in detail separately.

Measurements given in [29] indicate that the current wave form has a considerable influence on the gas phase emitter effect. Lower average electrode temperatures were found with a sinusoidal current than with a switched dc current of the same effective value. The influence of the different current wave forms will be investigated in detail in a subsequent paper.

\section{Concluding remarks}

The measurements at a YAG lamp seeded with $D y I_{3}$ confirm the initial speculation that a reduction of the work function by a gas phase emitter effect within the cathodic half period may overlap onto the anodic half period at higher operating frequencies. The condition is a sufficiently high lifetime of the atomic monolayer being formed by the emitter material. The lifetime depends among others on the intensity of the cataphoretic transport of ionized emitter material towards the cathode, the adsorption/desorption 
energy of the emitter atoms on the electrode surface and the temperature of the electrode surface. The cataphoretic transport of emitter material is in addition sensitively dependent on the gas composition as the example of a seeding with $D y I_{3}$ and $T l I$ has shown. Therefore it may be a difficult task to optimize the emitter effect in commercial MH lamps seeded by a mixture of metal iodides.

\section{Acknowledgements}

The authors thank Philips Lighting, NL, for scientific and financial support. Financial support from Deutsche Forschungsgemeinschaft (Graduiertenkolleg 1051) and from the Research School of the Ruhr-University of Bochum is also gratefully acknowledged by the authors.

\section{References}

[1] Lichtenberg S et al 2002 J. Phys. D: Appl. Phys. 35 1648-1656

[2] Lichtenberg S et al 2005 J. Phys. D: Appl. Phys. 38 3112-3127

[3] Dabringhausen L et al 2005 J. Phys. D: Appl. Phys. 38 3128-3142

[4] Benilov M S et al 2002 J. Phys. D: Appl. Phys. 35 1-15

[5] Waymouth J F 1971 Electric Discharge Lamps, Monographs in Modern Electrical Technology (Cambridge, MA: MIT Press)

[6] Dabringhausen L et al 2002 J. Phys. D: Appl. Phys. 35 1621-1630

[7] Luhmann J et al 2002 J. Phys. D: Appl. Phys. 35 1631-1638

[8] Nandelstaedt D et al 2002 J. Phys. D: Appl. Phys. 35 1639-1647

[9] Redwitz M et al 2006 J. Phys. D: Appl. Phys. 39 2160-2179

[10] Mentel J and Heberlein J 2010 J. Phys. D: Appl. Phys. 43023002

[11] Westermeier M et al 2010 J. Phys. D: Appl. Phys. 43124015

[12] Luijks G M J F et al 2005 J. Phys. D: Appl. Phys. 38 3163-3169

[13] Almanstoetter J et al 2002 J. Phys. D: Appl. Phys. 35 1751-1756

[14] Hartmann T et al 2010 J. Phys. D: Appl. Phys. 43025201

[15] Tielemans P and Oostvogels F 1983 Philips J. Res. 38 214-223

[16] Langenscheidt O et al 2008 J. Phys. D: Appl. Phys. 41144005

[17] Luijks G M J F et al 2008 J. Phys. D: Appl. Phys. 41144006

[18] Miedema A R and Dorleijn J W F 1980 Surface Science 95 447-464

[19] Medvedev B K, Ionov N I and Belyakov Yu I 1974 Sov. Phys. Solid State 15 (9) 1743-1746

[20] Alekseev N I 1968 Soviet Physics - Technical Physics 12 (12) 1639-1642

[21] Westermeier M et al 2008 IEEE Transactions on Plasma Science 36 (4) 1176-1177

[22] Hartmann T et al 2002 J. Phys. D: Appl. Phys. 35 1657-1667

[23] Redwitz M et al 2005 J. Phys. D: Appl. Phys. 38 3143-3154

[24] Langenscheidt O et al 2007 J. Phys. D: Appl. Phys. 40 415-431

[25] Reinelt J et al 2008 J. Phys. D: Appl. Phys. 41144002

[26] Benilov M S 2008 J. Phys. D: Appl. Phys. 41144001

[27] Benilov M S et al 2005 J. Phys. D: Appl. Phys. 38 3155-62

[28] Reinelt J et al 2011 J. Phys. D: Appl. Phys. 44095204

[29] Reinelt J 2009 Experimental investigation on electrodes of HID lamps at low and high operating frequencies PhD thesis, Ruhr University Bochum

[30] Dabringhausen L 2004 Charakterisierung von Elektroden für Hochdruck-Plasmalampen durch Pyrometrie und Simulation PhD thesis (Berlin Tenea) Ruhr Universität Bochum 
[31] DeVos J C 1954 Physica 20 690-714

[32] Westermeier M et al 2010 Phase resolved Dy-density and plasma temperature measurements by absorption- and emision-spectroscopy of Dy spectral lines, Proc. 12th Int. Symp. on the Science and Technology of Light Sources and the 3rd Int. Conf. on White LEDs and Solid State Lighting (Eindhoven (NL), 11-16 July 2010) ed Haverlag M et al CP060, 165-166

[33] Schoepp H 2002 Elektrische und strahlungsphysikalische Eigenschaften von Hochdruckentladungen - insbesondere Quecksilberdampfentladungen - für die Beleuchtungstechnik (Berlin: Technische Universität)

[34] Kurucz R et al 1995 KURUCZ CD-ROM NO. 23 - Atomic Line List (Cambridge, MA: HarvardSmithsonian Center for Astrophysics)

[35] Bergner A et al 2010 Temperature measurements at thoriated cathodes in a model lamp and its interpretation by numerical simulation, Proc. 12th Int. Symp. on the Science and Technology of Light Sources and the 3rd Int. Conf. on White LEDs and Solid State Lighting (Eindhoven (NL), 11-16 July 2010) ed Haverlag M et al CP128, 373-374

[36] Almeida N A et al 2009 J. Phys. D: Appl. Phys. 42045210

[37] Welters W Philips Lighting, GBU HID, P.O.box 80020, 5600JM Eindhoven, The Netherlands private communication 